\title{
Multiple Slot Allocation for Voice/Data Transmission over PRMA++ Applied to FRAMES Multiple Access Mode 1
}

\author{
Ramon Ferrús, Pilar Díaz \\ Department of Signal Theory and Communications (UPC) \\ C/ Jordi Girona 1-3 Campus Nord-Edifici D4 \\ 08034 Barcelona-Spain \\ Phone: +34-3-4015948 Fax: +34-3-401 7200 \\ e-mail: pilar, ferrus @xaloc.upc.es
}

\begin{abstract}
This paper presents some simulation results of PRMA++ for voice and data transmission over a physical air interface platform defined in the ACTS European project FRAMES. Variations on the statistics of speech sources (activity factor, petition rate) are studied and conclusions are obtained for optimal frame dimensioning. For data transmission, a multiple slot allocation scheme is presented and results are shown for different source rates and packet lengths.
\end{abstract}

\section{INTRODUCTION}

The third generation of wireless communications will be dominated by multimedia traffic. Considerable research effort has been invested to the design of multiple access schemes able to efficiently accommodate different rate requirements and quality of service over the same physical radio interface. Packet transmission seems to be better positioned than circuit-oriented transmission to accomplish the data transport layer issues even though the complexity of supporting real time services. PRMA+t is a multiple access protocol based on packet transmission. PRMA++ was proposed and studied within the RACE II European project ATDMA [1] to achieve, keeping the complexity at minimum, certain multiplexing statistic gain over circuit-oriented operation for basically voice transmission. The main difference of PRMA++ with the PRMA protocol [2] is the separation of the resources devoted to access, with contention, from the ones used to carry information, contention-free. Although PRMA has been proved to be very dependent on source statistics, this paper shows the benefits of using PRMA++ over a physical interface specification that it is currently under consideration in the European project FRAMES $[3,4]$. Voice and data transmissions have been analyzed and the capacity of multiple slot allocation for data traffic has been studied.

The choice of a TDMA access is motivated only by the intention of fixing a studied physical layer platform from which obtain performance results and it does not suppose any restriction to extend this application to different access technology. In fact, PRMA has been studied over CDMA [5] and further work is envisaged to analyze PRMA++ performance on a wideband CDMA packet transmission basis and to explode PRMA++ characteristics on a multicarrier configuration.

This paper is organized as follows. In section 2 the PRMA++ protocol is described. Section 3 deals with the radio interface considered in our study. In section 4 the voice transmission is analyzed. Firstly, the PRMA++ adaptation to the frame structure is described and then, optimal frame dimensioning is discussed taking into account variations on the statistics source, activity factor and petition rate. Finally section 5 deals with data transmission. A multiple slot allocation scheme is proposed and different source rates and packet lengths are considered. Conclusions close the paper in section 6 .

\section{PRMA+ DESCRIPTION}

The PRMA++ protocol is an augmented version of an ALOHA reservation protocol proposed in [6]. For PRMA++ the time is organized in frames that consist of time slots. In the uplink the mobiles ask for a reservation by means of a specific access packet but only in a certain number of slots named Reservationslots, R-Slots. After correct reception in the base station, an Information-Slot, I-Slot, is assigned to this mobile. The notification of the assignments is done using specific slots in the downlink named Acknowledge-Slots, A-Slots. The R and ASlots are paired and the A-slots follow the R-slots in time, offset by two-timeslot duration to allow for resource allocation processing delay. The management of the downlink channel is completely centralized, thus the base station notifies accordingly, by means of the Fast Paging Slots, to which I-Slots the mobiles must to listen to.

Adding information in the access packet useful for the base station to assign the I-slots efficiently could also optimize PRMA ++. This information could contain the capacity required, the type of service and even a status of the expiration time characteristics of the packets to be transmitted. Processing this information a single or multiple allocation could be done to satisfy the service requirements. The multiple assignation option is evaluated in the system level simulations for data services. 


\section{PHYSICAL AIR INTERFACE CONFIGURATION}

The FRAMES Multiple Access mode number 1, FMAl, has been chosen as physical layer to perform the system level simulations. FMAl is based on wideband TDMA with and without spreading. The users are separated orthogonally into time slots, and within each time slot, an additional separation by spreading codes could be used. The TDMA frame length is $4.615 \mathrm{~ms}$, as in GSM, and it could consist of $1 / 64,1 / 16$ and $1 / 8$ slots fitting together. The spreading capability is only used in the $1 / 8$ slots and it is not considered in this paper. The carrier symbol rate is $2.6 \mathrm{Msymbols} / \mathrm{s}$ and the modulation could be Binary Offset QAM or Quaternary Offset QAM depending on the link quality. The $1 / 64$ slots are able to transmit $144 / 122$ useful symbols per frame while the 1/16 slots support 684 symbols. A basic physical channel consists of one time slot in the case of FMAl without spreading, and user bit rates from a few kbit/s up to $2 \mathrm{Mbits} / \mathrm{s}$ could be achieved by allocating different number of basic physical channels. FMAl can be operated in FDD and TDD mode with the only restriction that in the TDD mode the minimum length of uplink and downlink parts is one eighth of the frame length. A single FMA1 carrier operated in FDD mode has been chosen in order to obtain the results presented in the following sections.

\section{SYSTEM LEVEL PERFORMANCE FOR VOICE TRANSMISSION}

A basic channel for mapping a speech service in a FMA1 carrier may consist of using a single $1 / 64$ slot per frame. With a modulation BOQAM and a code rate of 0.5 , this channel could support perfectly a 13kbits/s speech transmission. Even though in FMA1 the possibility of assigning a single slot each certain number of frames is considered, for example 0.5 slots per frame to carry a $8 \mathrm{kbits} / \mathrm{s}$ speech service, we do not include this particularity in order not to increase the complexity of the simulation program and to allow PRMA++ to work on a frameby-frame basis.

We consider a complete carrier only devoted to carry speech information to show the benefits of using a simple reservation mechanism in front of having circuit-oriented channels. To implement the PRMA++ protocol several 1/64 slots within the frame must be used as access slots (R-Slots). The rest are assigned by the base station to the active mobiles after receiving the corresponding notification in the R-Slot . The packet access contains a temporal identity of the mobile and a FIFO schedule policy is applied in the base station. The downlink channel is not considered for the speech simulations since the limitations are found in the uplink due to the reservation process. To simulate the A-siots a delay of two time slots is introduced before transmitting the allocations or just the acknowledgments for correct reception in the R-Slots. The maximum allowable delay for speech packet delivery has been set to $10 \mathrm{~ms}$.

\section{A. Effect of the retransmission probability.}

To achieve a high multiplexing gain with PRMA+t it is important to have the maximum resources devoted to carry useful information without limiting the random access capacity. Collisions in the access channel are solved by a retransmission mechanism according to a parameter broadcasted from the base station. This retransmission probability has an important influence on the global performance as it is shown in figure 1. So it seems necessary to calculate this value in a slot by slot basis taken into account the load status. Techniques developed to stabilize a slotted-aloha channel are valid here, i.e. the pseudo Bayesian algorithm [7].

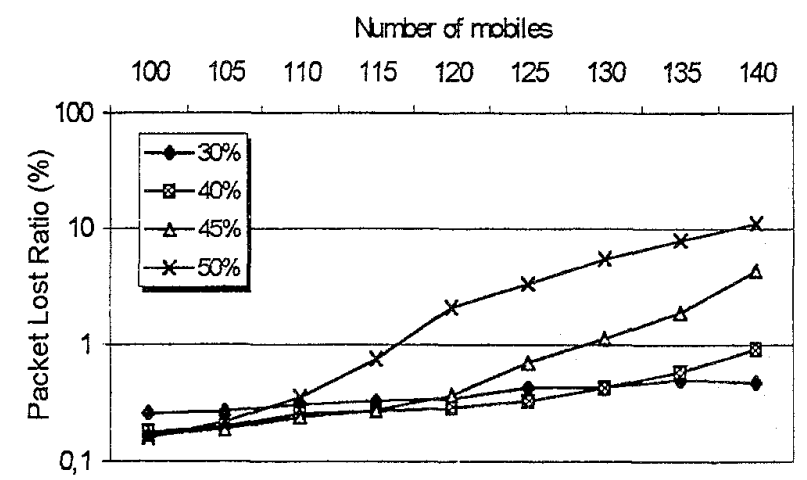

Figure 1. Effect of the retransmission probability (100 slots/frame with one R-Slot.

We have adopted the following expression for the adaptation of the retransmission probability. It is based on an estimation of the number of mobiles in retransmission state from the previous number of collisions in the access channel.

$$
p r=\min \left(1,\left(1-r^{*}(\mathrm{M}-R-E R)\right) / E R\right)
$$

where

$\mathrm{r}=$ Petition generation rate per mobile unit (Estimated statistically).

$\mathrm{M}=$ Total number of active mobiles.

$\mathrm{R}=\mathrm{M}$ obiles in reservation mode.

$E R=$ Estimated number of retransmissions.

Next table shows the resulting packet error ratio versus active mobiles with the same configuration considered in figure 1.

Table I. Effect of calculating pr adaptatively in a slot-by-slot basis.

\begin{tabular}{|l|l|l|l|l|l|l|l|l|l|}
\hline MOBLES & 100 & 105 & 110 & 115 & 120 & 125 & 130 & 135 & 140 \\
\hline
\end{tabular} \begin{tabular}{|l|l|l|l|l|l|l|l|l|l|}
\hline PLR(\%) & 0,18 & 0,18 & 0,20 & 0,23 & 0,26 & 0,26 & 0,31 & 0,32 & 0,35 \\
\hline
\end{tabular} 


\section{B. Optimal Frame dimensioning.}

The optimum number of R-Slots necessary in the frame for voice reservation depends mainly on the statistic of the voice sources. A commonly agreed model to generate speech traffic is a two-state Markov chain [8]. Also the mean duration in the silence period and in the active period of $1.74 \mathrm{~s}$ and $1.41 \mathrm{~s}$ respectively are often used [9]. This yields an activity factor of $45 \%$. In figure 2, Packet Lost Ratio is shown in terms of the number of R-slots for several load conditions. When there are few mobiles the packet loss is due mainly to the access time. When the traffic load increases the limiting factor for packet loss becomes the assignation time because it is more difficult to find a free slot.

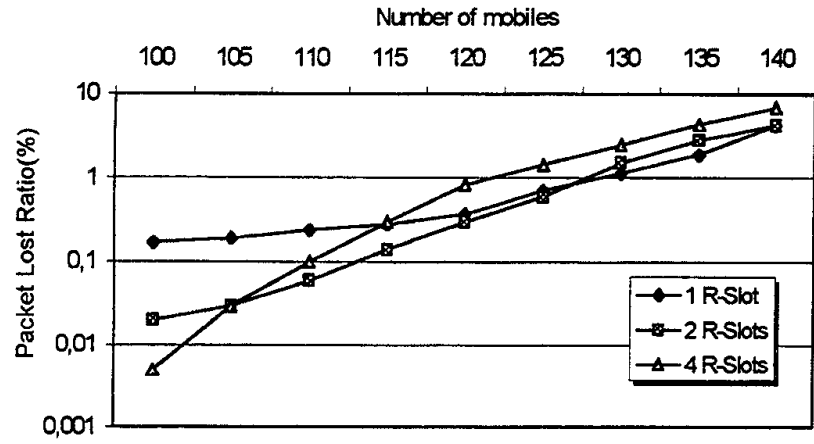

Figure 2. Packet Lost Ratio for different R-Slots configurations.

The best configuration results in an adaptive number of R-Slots based on the number of active mobiles in the system. From figure 2 we observe that only with 1 R-slot, PLR could be kept below $1 \%$ with relatively high load. It is important to remark the multiplexing gains over a circuit-switched operation mode obtained with whenever an adaptive or static R-Slot configuration. Evidently, more studies taken into account the error probability and the capture effect on the access channel have to be performed, but the values in table II give us an approximate idea of the benefit of using a PRMA++ protocol in one FMA1 carrier only devoted to carry speech traffic.

Table II. Multiplexing gain achievable over a circuit-switched configuration maintaining a packet error ratio less than $1 \%$.

\begin{tabular}{|l|l|l|l|l|l|l|l|}
\hline Mobiles & 100 & 105 & 110 & 115 & 120 & 125 & 130 \\
\hline $\begin{array}{l}\text { Gain } \\
\text { PRMA++ }\end{array}$ & 1,56 & 1,64 & 1,72 & 1,79 & 1,87 & 1,94 & 2,01 \\
\hline
\end{tabular}

\section{Effect of the activity factor.}

In this section we have analyzed the dependence of the previously found values with variations in the speech activity factor. Figure 3 shows that the PLR depends strongly on the activity factor considered and consequently the maximum number of simultaneous users the system can support. As expected, when the activity factor increases the PLR becomes worse in high load conditions and this tendency is even more accused by increasing the number of access slots. For an activity factor of $50 \%$ the system supports approximately 20 users less simultaneously than for a factor of $45 \%$. Meanwhile for activity factors below $40 \%$ the system capacity increases considerably allowing 140 speech users to be supported simultaneously with a packet loss lower than $1 \%$.

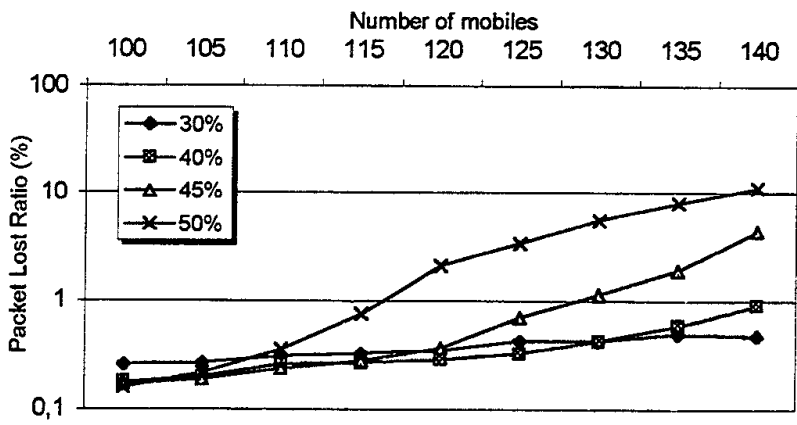

Figure 3. Effect of the speech activity factor on PLR (1 R-Slot)

\section{Effect of the absolute values in Ton, Toff.}

Apart from the speech activity factor it is also interesting to evaluate the dependence of the performance with the absolute values for the mean duration periods of activity and silence. The inverse of thie period Ton+Toff could be seen as the rate at which petitions are generated to ask for resources. The figure 4 shows the effect of varying this rate maintaining fixed the activity factor to $45 \%$. The value 0.3 is obtained by inverting the sum of Ton and Toff considered in 4.2 . The value 0.2 corresponds to Ton $=2.23$ and Toff $=2.76$ while the value 0.4 is obtained from Ton=1.11 and Toff $=1.38$. We have only represented the $1 \mathrm{R}$-slot configuration since with $2 \mathrm{R}$-Slots or more the performance remains quite constant. It is also interesting to observe that really the effect only is appreciable in the load region where the packet losses are due to access time.

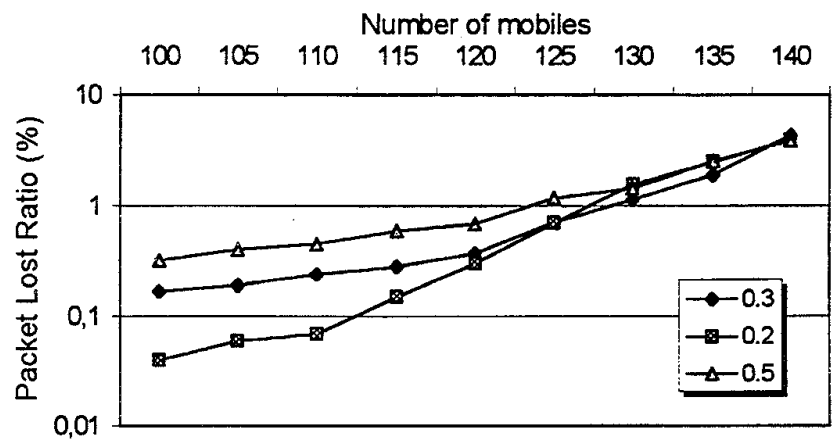

Figure 4. Effect of the petition rate (1 R-Slot) 


\section{SYSTEM LEVEL PERFORMANCE FOR DATA TRANSMISSION}

Most results for PRMA++ operation are basically pointed to speech transmission. In this section we show some results for packet data transmission managed by a PRMA++ protocol. As in voice transmission, we consider a single FMAl carrier to analyze performance parameters. For data transmission we allow the system to make multiple slot allocations depending on a priority value transmitted in the access burts and also based on the load conditions.

The data sources considered are modeled using a Poisson process and quantified by means of a factor, $\alpha$, representing the percentage of the maximum transmission rate that a single 1/64 slot assigned in circuit-switched operation can support. With an OQAM modulation and a code rate of about $0.5 \mathrm{a}$ basic rate of $32 \mathrm{kbit} / \mathrm{s}$ is taken as reference. Our interest is to characterize a scenario with more active mobiles than resources and so the values considered for $\alpha$ are below 1 .

To simulate a bursty traffic the data sources generate messages of constant length. Four different lengths have been chosen to evaluate the performance of the protocol. Concretely messages of $32,128,512$ and 2048 bytes are taken into account and to transmit them it is necessary to use $2,8,32$ or 128 physical $1 / 64$ bursts respectively.

In multiple slot allocation mode the base station tries to assign firstly one $1 / 16$ slot, that is approximately equivalent to four $1 / 64$ slots. The allocation is successfully done in case of having less occupied slots than a maximum ( $M a x$ numl). If the allocation is not possible, the base station tries to assign two $1 / 64$ slots evaluating now the condition for the busy slots not to exceed a new maximum (Mox_num2). Finally, if neither $1 / 16$ slot nor two 1/8 slots have been allocated, the base station tries to assign a slot as in single allocation mode.

\section{A. Simulation results}

Results have been obtained for data sources with mean generation rate of $16 \mathrm{kbps}(\alpha=0.5)$ and $24 \mathrm{kbps}(\alpha=0.75)$. The values used to manage the multiple allocation, Max_num I and Max_num2, have been set to MaxRes-16 and Maxres- 8 respectively, where MaxRes is the maximum number of slots dedicated to carry useful information.

Figures 5 and 6 show the message transfer delay for packets of length 128 bytes for sources with transmission rate of $16 \mathrm{kbps}$ and $24 \mathrm{kbps}$ respectively. In both cases we observe that the $8 \mathrm{R}$ slots configuration for the access channel performs better. That is due to the fact that, as the packet lengths are shor, many petitions have to be generated to acquire resources and so the capacity provided by the access channel fixes basically the message delay. Furthermore in both cases the multiple allocation scheme with two R-slots worsens the transfer delay since the petition rate increases considerably. With eighth Rslots the improvement of multiple allocation is practically negligible. We can also observe that when the load increases the system is limited by the mean allocation time, performing better as less number of R-slots. The throughput achieved could reach above $85 \%$, i.e. 100 mobiles with $\alpha=0.5$, without loosing packets and maintaining a reasonable message transfer delay.

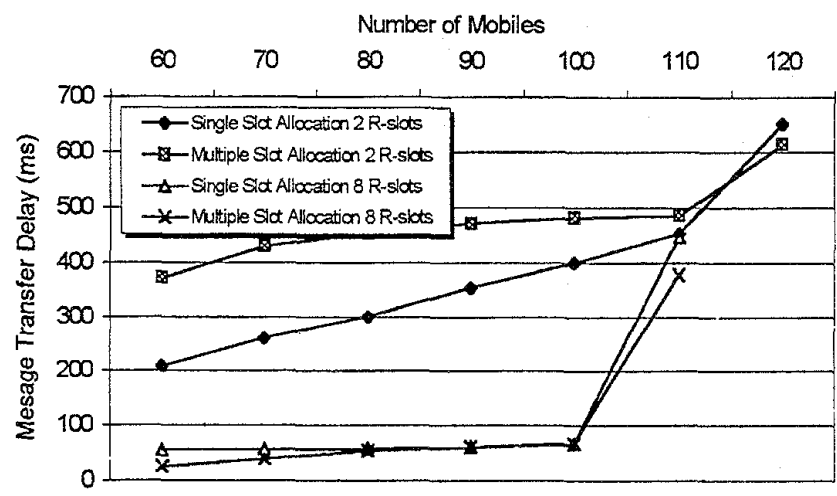

Figure 5. Delay for 128 bytes message length and $16 \mathrm{kbps}$ sources.

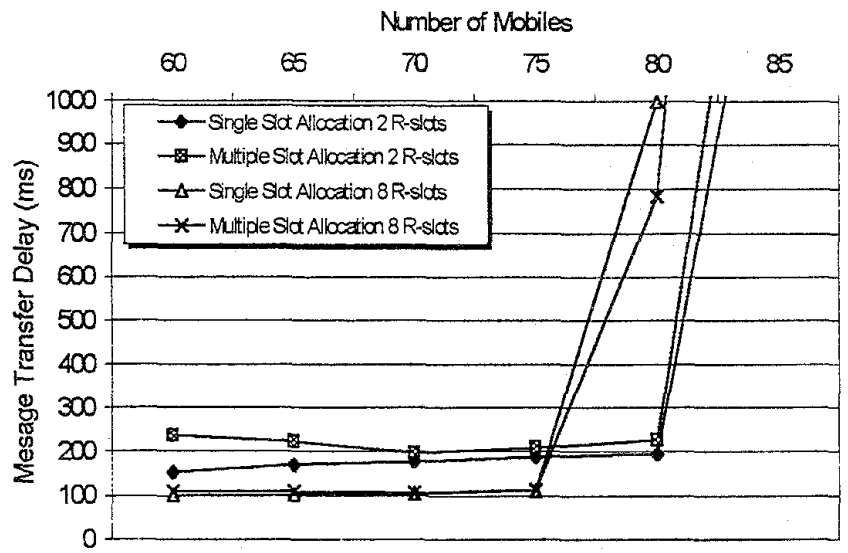

Figure 6. Delay for 128 bytes message length and $24 \mathrm{kbps}$ sources.

Figures 7 and 8 are analogous to the previous ones but assuming a message length of 2 kbytes. The mean generation rate of the sources has been kept constant. Now we observe that in medium load conditions, for long message lengths, the multiple allocation improves considerably the message transfer for any number of R-slots. Even the behavior now is better for the two-R-slots configuration because the petition rate is much lower than for 128 bytes messages and the system is not limited by the access time. For $24 \mathrm{kbps}$ sources the improvement is minimum because of the system is always too loaded to be able to assign multiple slots. The throughput achieved with long messages are even higher than the previous cases because the 
number of R-slots needed is minimum, i.e. 120 mobiles of $16 \mathrm{kbps}$ could be supported with message transfer delay of about 1 second.

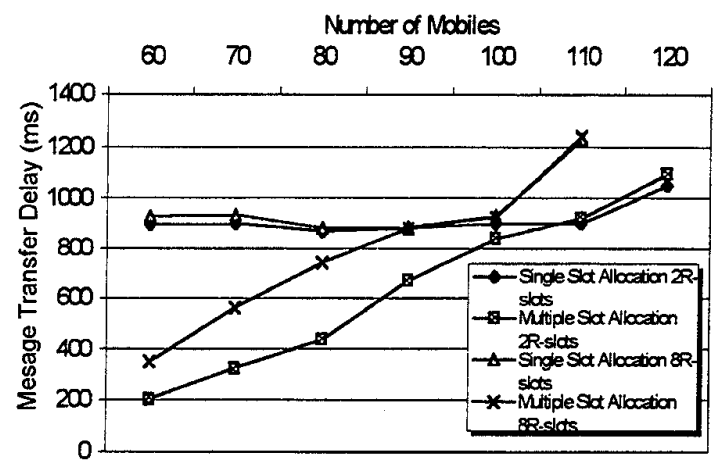

Figure 7. Delay for 2 kbytes message length and $16 \mathrm{kbps}$ sources.

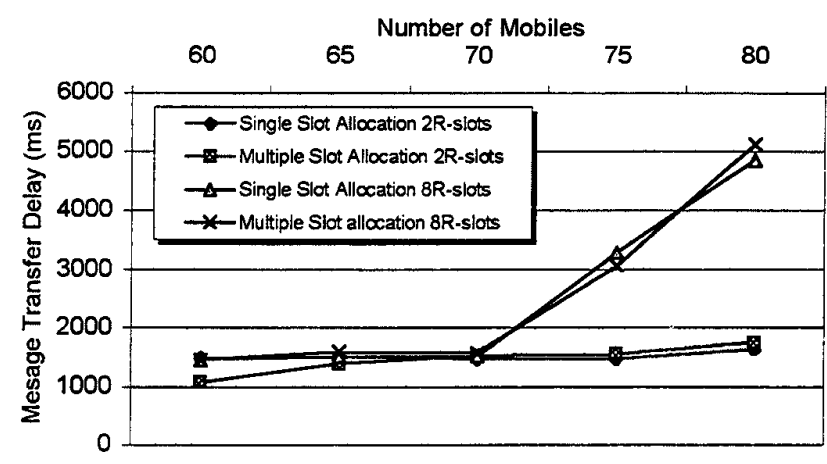

Figure 8. Delay for 2 kbytes message length and $24 \mathrm{kbps}$ sources.

\section{CONCLUSION}

We have analyzed PRMA++ for speech traffic applied to a physical configuration under consideration on the European project FRAMES. Even though the dependence of the performance parameters obtained with PRMA++ on the statistics of the sources, the effect of the activity factor and petition rate have been studied, it is shown that a high multiplexing gain, over a circuit-oriented mode, could be achieved without introducing too much complexity.

PRMA++ for data transmission also have been evaluated. Maintaining the same physical configuration, we show the behavior for different source rates, $16 \mathrm{kbps}$ and $24 \mathrm{kbps}$, and different messages lengths, 32bytes, 128 bytes, 512bytes and $2 \mathrm{kbytes}$. Apart from dimensioning the number of R-slots, a multiple slot allocation mode has been studied giving some improvement for medium load conditions and long packets.

Further studies will consider the performance for mixed data traffic, including different priority levels for the multiple scheme allocation. Also in case of very short length packets, the possibility of adding this information in the access packet could be envisaged. Finally, the application of this kind of access scheme to a wideband CDMA system is also object of study.

\section{REFERENCES}

[1] ATDMA Project, "Radio Network Control Techniques", ATDMA Deliverable R2084/RMR/TS3/DS/L/040/bl, January 1995.

[2] D.J. Goodman, R.A. Valenzuela et al., "Packet Reservation Multiple Access for Local Wireless Communications", IEEE Trans. on Communications, vol. $37, \mathrm{n}^{\circ} 8, \mathrm{pp} 885-890$, August 1989.

[3] Eero Nikula et al.,"Flexibility in Providing UMTS Services with FRAMES FMAI", ACTS Mobile Communications Summit, Aalborg, October 97.

[4] Fredrik Ovesjö et al., "Frames Multiple Access Mode 2Wideband CDMA", Proc. IEEE Int. Conf. On Personal Indoor and Mobile Radio Commun., PIMRC'97, Helsinki, Finland, September 1997.

[5] Alex E. Brand and A. Hamid Aghvami, "Performance of a Joint CDMA/PRMA Protocol for Mixed Voice/Data Transmission for Third Generation Mobile Communication", IEEE Journal on Selected Areas in Communications, vol. 14, n 9, December 1996.

[6] N.M. Mitrou, TH. D. Orinos and E.N. Protonotarios, "A reservation Multiple Access Protocol for Microcellullar Mobile-Communication system", IEEE Trans. on Vehicular Technology ., vol. 39, n4, pp 340-351, November 1990.

[7] R.L.Rivest, "Network Control by Bayesian Broadcast", IEEE Trans. on Information Theory, Vol IT-33, $\mathrm{n}^{\circ} 3$, pp 323-328, May 1987.

[8] D.J. Goodman, S.X.Wei, "Efficiency of Packet Reservation Multiple Access", IEEE Trans. on Vehicular Technology, vol. $40, \mathrm{n}^{\circ} 1$, pp 170-176, February 1991.

[9] John Dunlop, James Irvine, David Robertson and Peter Cosimini, "Performance of a Statistically Multiplexed Access Mechanism for a TDMA Radio Interface", IEEE Personal Communications, June 1995. 